\title{
REGIONAL DIFFERENCES IN THE NEURONAL CYTOSKELETON $^{1}$
}

\author{
PETER F. DRAKE AND RAYMOND J. LASEK ${ }^{2}$ \\ Department of Developmental Genetics and Anatomy, Case Western Reserve University, School of Medicine, Cleveland, Ohio \\ 44106
}

Received May 16, 1983; Revised August 29, 1983; Accepted August 30, 1983

\begin{abstract}
Regional differences in the neuronal cytoskeleton were investigated in the giant neurons of Aplysia. Using SDS-PAGE, we have compared the proteins which comprise the cytoskeletons of cell bodies and axons. Separate populations of cell bodies and axons were collected and the proteins stained by the Coomassie brilliant blue method. Individual identified cell bodies, with long segments of their axons attached, were isolated, and the proteins were labeled with the $\left.{ }^{125} \mathrm{I}\right]$ Bolton-Hunter reagent. The proteins which are stably associated with the cytoskeleton were obtained by extracting the neuronal material in a physiological buffer containing Triton X-100. As a correlative measure to the biochemical analyses, electron microscopy was performed on the cell body and axonal fractions.

Our results demonstrate that the composition and biochemical properties of the cytoskeletal proteins in the neuron cell bodies differ from those associated with axons. Specifically, the amount of neurofilament proteins, designated NF60 and NF65, is 5 times more abundant in the axon than in the cell body. The relative amounts of actin and tubulin are comparable in these two regions of the neuron. In addition, the ratio of NF60 and NF65 is different in the cell body and axon. The cell bodies contain proportionally more NF60 than the axons. However, the physical properties of the tubulin in the cell body, as measured by relative solubility, differ from that of the axon. The substantial differences between the composition of the cytoskeleton of the cell bodies and axons of Aplysia suggests that at least two distinct cytoskeletal networks exist in these neurons, one specific for the cell body and the other specific for the axon.
\end{abstract}

Neurons exhibit a remarkable degree of regional differentiation in their external form. For example, the axons and dendrites of a particular neuron usually have completely different morphologies. These differences in morphology apparently reflect regional differences in the composition of the cytoskeletal elements that provide the architectural framework for the neuron. For example, electron microscopic studies demonstrate that in a variety of vertebrate neurons, including the Purkinje cells and basket cells, the ratio of microtubules to neurofilaments (MT/NF) is significantly higher in the dendrites than in the axons (Palay and Chan-Palay, 1974). Immunocytochemical studies also indicate that the detailed composition of the cytoskeleton differs within individual

\footnotetext{
${ }^{1}$ This research was supported by National Institutes of Health Grant NS-14900-03 to R. J. L. and National Institutes of Health Traineeship NS-07118-05 to P. F. D. We wish to thank Dr. Michael Katz and Dr. Monica Oblinger for helpful discussions and suggestions, and Ms. Shirley Rickets and Ms. Dianne Filsinger for excellent technical assistance throughout the work.

${ }^{2}$ To whom correspondence should be addressed.
}

neurons. For example, certain microtubule-associated proteins (the high molecular weight proteins, HMW) are associated preferentially with microtubules in dendrites and may not be present in axons (Matus et al., 1981). Axonal transport studies indicate that the $\tau$ proteins are the major microtubule-associated proteins of the axon (M. Tytell, S. Brady, and R. Lasek, submitted for publication).

These regional differences in the cytoskeleton raise the possibility that the neuronal cytoskeleton can be divided into specialized regions each with its own organizational determinants. It would clearly be of value to develop experimental models in which the regional differentiation of the cytoskeleton could be studied directly with biochemical methods. The giant neurons of Aplysia provide an opportunity to compare the cytoskeletal proteins of the cell body with those in the axon of individual identifiable cells. These neurons have a relatively simple geometry. Like most molluscan neurons, Aplysia neurons are unipolar and give rise to a single axon which branches into both the receptive neurites and effector neurites. 
Molluscan neurons do not produce dendrites directly from the cell body. Instead, the axons subserve the functions that both axons and dendrites serve in vertebrates (Kandel, 1976).

Despite the apparent morphological simplicity of molluscan neurons, they may possess two regionally distinct cytoskeletons, one which is associated with the axon and another which is limited to the cell body. In fact, it has been proposed that the segregation of the axonal cytoskeleton from that of the cell body may be one of the crucial developmental steps that distinguishes neurons from other migratory cell types (Lasek, 1982). Neurons differ from other migratory cells with regard to their mode of locomotion (Lasek, 1981). Most cells move by extending their leading edges and towing their cell bodies behind them, but neurons can extend their axons without towing the cell body and all of its contents behind the growing axon. This ability to translocate the axonal cytoskeleton without towing the perikaryon suggests that the axonal cytoskeleton is organized separately from the cytoskeleton of the cell body.

To evaluate the possibility that the cytoskeleton within the cell body differs from that within the axon, we have compared the proteins which comprise the cytoskeleton in cell bodies and axons from the central nervous system of Aplysia. Cell bodies are easily obtained from the ganglia of Aplysia because the neurons are located in the cortical rind, and cells are separated from each other by a thin layer of glial cells. Preparations enriched with axons can be obtained from the central nervous systcm by desheathing the neural connectives that course between the ganglia. We have also developed a technique whereby individual identified neurons are dissected from the ganglia with a long segment of their axons attached. Using this technique, it is possible to compare the proteins in the cell body with those in the axon from a single identified neuron. Furthermore, the nucleus can be removed from the cell body so that a preparation principally consisting of cytoplasm is obtained.

It is possible to analyze selectively the proteins which are stably associated with the cytoskeleton by permeabilizing cells with detergents and then extracting the cells in a buffer solution which preserves the cytoskeletal architecture. This method yields two distinct fractions: (1) a Triton-soluble ( $\mathrm{Ts}$ ) fraction which contains proteins that can diffuse from the permeabilized cells; and (2) a Triton-insoluble (Ti) fraction which contains proteins that remain tightly associated with the cell remnant. The Ts fraction contains cytoskeletal proteins that are either in the form of monomer or the form of polymers which disassemble readily when the cells are permeabilized. These proteins diffuse into the Ts fraction along with the other soluble proteins of the cell. By contrast, the Ti fraction contains those cytoskeletal polymers that remain stably polymerized during the extraction period. The relative proportion of cytoskeletal proteins which enter the Ts or Ti fractions is influenced by the solution conditions. Some buffers will tend to stabilize the cytoskeletal elements and others will solubilize the cytoskeleton. The buffers employed in this study have previously been found to maintain a fraction of the cytoskeleton in the polymerized state (Morris and Lasek, 1982). The proteins in the $\mathrm{Ti}$ and $\mathrm{Ts}$ fractions were analyzed by either one- or two-dimensional SDS polyacrylamide gel electrophoresis (SDS-PAGE)

\section{Materials and Methods}

Cell body-axon isolation. Aplysia californica, weighing 150 to $200 \mathrm{gm}$, were purchased from Marine Specimens Unlimited (Pacific Palisades, CA). The animals were maintained at $17^{\circ} \mathrm{C}$ in individual chambers containing circulating artificial sea water (Instant Ocean, Aquarium Systems Inc., Eastlake, OH).

The central nervous system of Aplysia was removed through an incision in the foot. To obtain populations of cell bodies for this study, central nervous system ganglia were pinned through the connective tissue sheath in a dish lined with Sylgard (Dow Corning) and containing a stabilizing buffer (buffer B or phosphate stabilizing buffer, see below). The connective tissue sheath lying over the cell bodies was removed by microdissection, and individual cells were plucked out using microforceps. If the microforceps were placed around the base of the cell body at the sight of axonal emergence, and the cell body was slowly pulled from the ganglion, it was possible to isolate a cell with a long segment (greater than $500 \mu \mathrm{m}$ ) of its axon attached (Fig. 1C).

For analysis by the Coomassie brilliant blue (CBB) staining method, a pooled fraction of individual isolated abdominal ganglion cell bodies (approximately 10 cells), free of axons and adherent neuropil, was collected. In addition, a pooled fraction enriched in axons was obtained by desheathing the long $(4 \mathrm{~cm})$ pleural abdominal connectives. This was accomplished by transecting the connective sheath layer with a sliver of razor blade while holding the axon bundle with microforceps, then peeling the entire sheath away (see Fig. $1 D$ ).

Using these cell body and axon isolation techniques, three cellular fractions were obtained (1) single isolated cell bodies, (2) single cell bodies with long segments of their axons attached, and (3) a pooled fraction enriched with axons.

Radiolabeling polypeptides using the Bolton-Hunter reagent. The Bolton-Hunter reagent is the iodo- form of $N$-succinimidyl 3-(4-hydroxy, phenyl)propionate which has been used successfully to label proteins in a variety of systems to high specific activity (Bolton and Hunter, 1973; Katz et al., 1982). The Bolton-Hunter reagent readily crosses cell membranes and, therefore, can be applied extracellularly to label intracellular proteins. Moreover, this reagent does not appear to have deleterious effects on living tissue as it has been used to study biological systems ranging from developing frog embryos (Katz et al., 1982) to in vivo axonal transport (Fink and Gainer, 1979, 1980). The utility of this agent for our study is that it increases the sensitivity of the analytic methods used and allows the study of proteins in distinct regions of a single neuron.

Diiodinated $\left[{ }^{125} \mathrm{I}\right]$ Bolton-Hunter reagent $(10 \mathrm{mCi} / \mathrm{ml}$, New England Nuclear) was supplied in benzene. Aliquots containing $100 \mu \mathrm{Ci}$ of the reagent were removed using a Hamilton syringe and were air dried on single depression glass microscope slides. Immediately after the benzene 

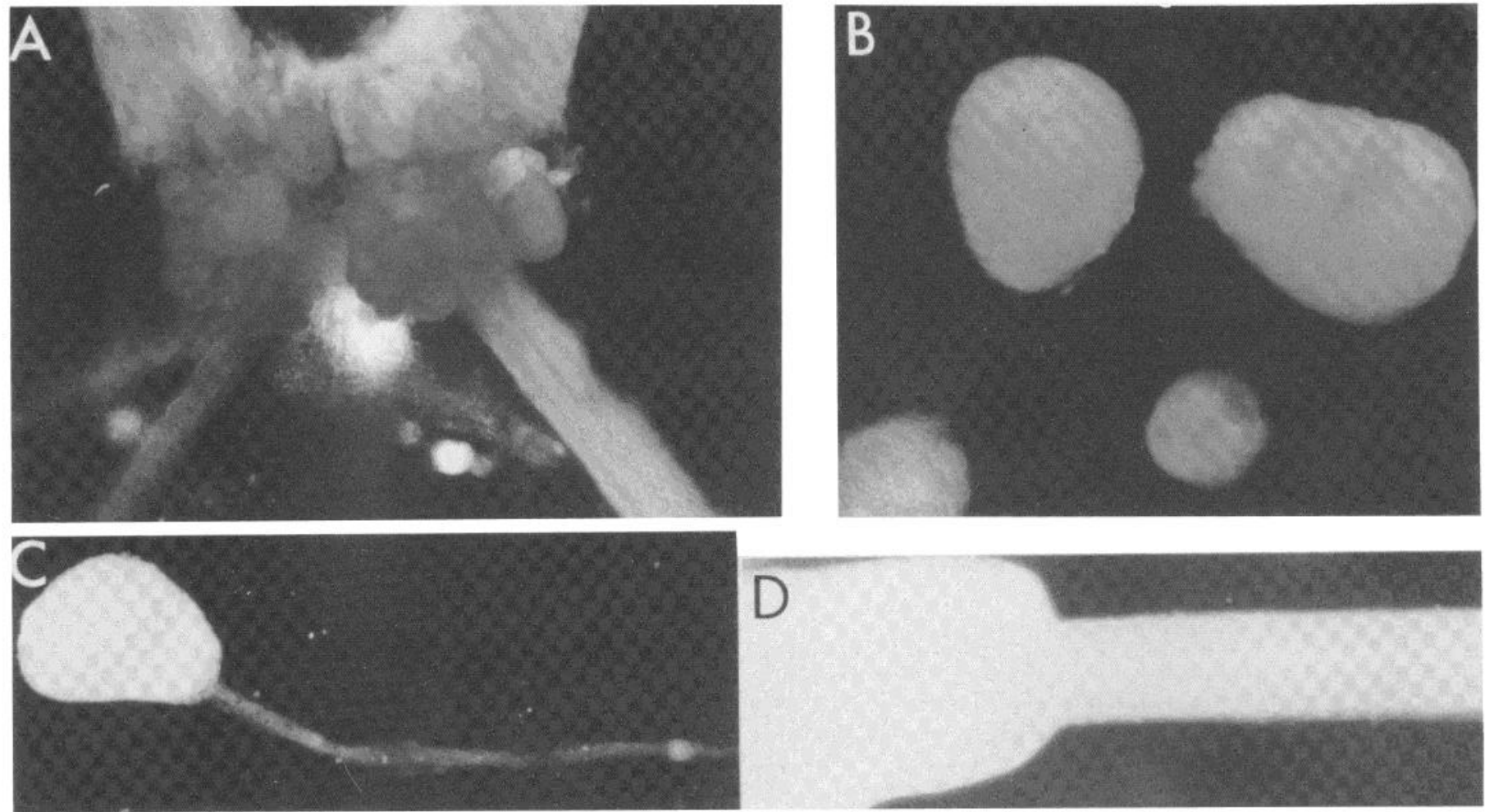

Figure 1. Topography of abdominal ganglion and isolated neurons. A, Excised abdominal ganglion with cortically located cell bodies. Magnification $\times 25$. $B$, Isolated giant neurons free of adherent tissue . Magnification $\times 50$. $C$, Isolated neurons with segment of proximal axon attached. Magnification $\times 50 . D$, Partially desheathed axon-containing connectives. Magnification $\times$ 25 .

evaporated, the cells to be labeled were added in a 100 $\mu \mathrm{l}$ aliquot of phosphate stabilizing buffer. To label TS proteins, the Bolton-Hunter reagent was dried in plastic centrifuge tubes, after which the entire volume of extracted proteins was added. These proteins were subsequently acid precipitated as described below. The reaction was allowed to proceed for $30 \mathrm{~min}$ at room temperature, after which the cells were removed and washed repeatedly in a buffer containing excess glycine. For further details regarding experimental techniques, see Appendix, Katz et al. (1982).

All cellular fractions destined for labeling with the Bolton-Hunter reagent were dissected and/or detergent extracted in phosphate-containing cytoskeletal stabilizing buffer. When fractions were to be stained with CBB, dissection and/or detergent extraction was performed in buffer B.

Preparation of the perikaryal and axonal cytoskeleton. Cytoskeletal fractions were prepared by extracting cellular fractions in $1 \mathrm{ml}$ of buffer B (after Rubinson and Baker, 1979; $300 \mathrm{~mm}$ potassium methanesulfonate, 150 $\mathrm{mM}$ taurine, $100 \mathrm{mM}$ potassium glutamate, $12.9 \mathrm{mM}$ $\mathrm{MgCl}_{2}, 10 \mathrm{mM}$ EGTA, $20 \mathrm{~mm}$ MOPS, and $1 \mathrm{~mm}$ GTP, pH 7.2) containing $1 \%$ Triton X-100 at room temperature for $2.0 \mathrm{hr}$. This buffer was developed to simulate the solution conditions of axoplasm. The resulting $\mathrm{Ti}$ fraction has been operationally defined as the stable cytoskeletal fraction. The Ts fraction was precipitated with trichloroacetic acid (TCA, final volume $10 \%$ ), centrifuged at $20,000 \mathrm{rpm}$ for $30 \mathrm{~min}$ and the pellet was washed with ether/ethanol (1:1) and ether.
$S D S-P A G E$. Samples to be electrophoresed were solubilized in either BUST ( $2 \% \beta$-mercaptoethanol, $8 \mathrm{M}$ urea, $1 \%$ SDS, and $10 \mathrm{~mm}$ Tris, pH 6.8$)$ or SUB $(0.5 \%$ SDS, $8 \mathrm{~m}$ urea, and $2 \% \beta$-mercaptoethanol, $\mathrm{pH} 6.8$ ) for one- and two-dimensional SDS gel electrophoresis, respectively.

One-dimensional SDS gel electrophoresis was by the method of Laemmli (1970) on a slab gradient (6 to $17.5 \%$ using a $4 \%$ stacking gel). Two-dimensional gel electrophoresis was after the method of O'Farrell (1975) employing the standard range of ampholines (pH 5 to 7 , LKB Instruments, Inc.). Proteins were stained with $1 \%$ CBB as described in Laemmli (1970). When radioactive samples were electrophoresed, the gels were vacuum dried onto dialysis membrane and autoradiographed as in Bonner and Laskey (1974). Audioradiographs were made using Lightening Plus Intensifying Screens (DuPont) and XR-5 x-ray film (Kodak) at $-70^{\circ} \mathrm{C}$.

The cytoskeletal proteins tubulin and actin were identified by co-migration with purified squid brain tubulin and purified chicken gizzard actin on one- and twodimensional gels.

Densitometric quantitation of proteins separated by SDS-PAGE. Proteins separated by one-dimensional SDS-PAGE and stained with CBB were quantitated by densitometry. Successive gel columns from a slab gel were scanned using a Zeiss scanning densitometer (model M4Q III), and the areas under absorbance peaks (at 550 $\mathrm{nm}$ wavelength), representing polypeptides of interest, were measured using digitization planimetry. Digitization planimetry was performed with the aid of a Numon- 
ics model 1224 electronic digitizer. The proteins from unextracted neuronal samples wre expressed as a percentage of total protein in each gel column. The relative solubility of actin, tubulin, and the neurofilament proteins was measured as a percentage of the amount of each protein which remained associated with the $\mathrm{Ti}$ fraction, as compared to the amount of protein in the $\mathrm{Ti}$ and Ts fractions combined.

Preparation for electron microscopy. Neuronal samples were prepared for electron microscopy by fixing in a buffered $2.5 \%$ glutaraldehyde solution $(20 \mathrm{mM}$ phosphate buffer, $2 \mathrm{mM}$ EGTA, balanced to isotonicity with glucose). Fixed samples were treated with $1 \%$ osmium tetroxide before dehydration and embedding in spur. Sections were cut with a diamond knife and stained with uranyl acetate-lead citrate stain before examination in the electron microscope.

\section{Results}

\section{Cell and axon population studies}

SDS-PAGE comparison of unlabeled cytoskeletal proteins in populations of cell bodies and axons. The desheathed abdominal ganglion with its cortically located cell bodies and axon-containing connectives is shown in a light micrograph taken with a dissecting microscope (Fig. 1A). The cell bodies were isolated either free of adherent tissue or with a long segment of axon attached (Fig. 1, $B$ and $C$ ). Samples enriched with axons were obtained by desheathing the pleural-abdominal connectives (Fig. $1 D$ ). It was also possible to collect clean samples of the neuropil because the cell bodies are located as a rind surrounding the deeper neuropil which contains all of the synaptic connections of the ganglion. The proteins from each of these sources were analyzed by one-dimensional SDS-PAGE (Fig. 2). Comparisons between the cell bodies, axons, and neuropil indicate that there are similarities between the composition of these different regions of the neuron. Tubulin and actin are prominent proteins in all of these fractions. The cell bodies, axons, and neuropil also contain significant amounts of two proteins with approximate $M_{\mathrm{r}}=250,000$ and 240,000 . These latter proteins appear to be the molluscan form of brain spectrin (fodrin) because their two-dimensional eletrophoretic behavior is similar to that of authentic fodrin (Levine and Willard, 1981; Bennett et al., 1982). Furthermore, like fodrin these proteins have been found to be preferentially associated with the region underlying the plasma membrane in the squid giant axon (Baumgold et al., 1981). Although there are many similarities between the protein compositions of the cell bodies, axons, and neuropil, one major difference stands out. Two proteins with $M_{\mathrm{r}}=65,000$ and 60,000 are particularly prominent in the axon-enriched fraction but are present to a much lesser extent in the cell body and neuropil fractions (Table I).

The connective tissue sheath which was stripped off of the connectives has a completely different composition than the axonal fraction and the other fractions that principally consist of cytoplasmic proteins. The sheath has a relatively simple protein composition and primarily consists of actin and proteins with $M_{\mathrm{r}}=55,000,93,000$ and 200,000. The large amount of actin in the sheath is consistent with the presence of contractile cells in the sheath which can contract the connective. The 93,000dalton protein is probably collagen, and the 200,000dalton protein may be collagen dimer.

Triton-insoluble proteins from populations of cell bodies and axons. Preparations enriched in cytoskeletal proteins were prepared by extracting cell bodies or axons in a volume of buffer containing 1\% Triton X-100. This procedure yields a $\mathrm{Ti}$ fraction and a $\mathrm{T}$ s fraction. Analyses of these fractions on SDS-PAGE demonstrate that the Ts fractions are generally more complex than the $\mathrm{Ti}$ fractions (Fig. $2 B$ ). Actin and tubulin are found in both the $\mathrm{Ti}$ and $\mathrm{Ts}$ fractions. However, fodrin (250,000 daltons and 240,000 daltons) and the 60,000-dalton and 65,000dalton proteins are present almost exclusively in the $\mathrm{Ti}$ fraction. This is particularly apparent in the axon fractions, in which the 60,000 -dalton and 65,000 -dalton proteins are unquestionably the most abundant proteins. The abundance of the 60,000-dalton and 65,000-dalton proteins in the axonal fraction and their relative insolubility raised the possibility that these proteins were the neurofilament proteins of Aplysia.

This possibility is supported by the following observtions. Both the 60,000-dalton and 65,000-dalton proteins cross-react with antibodies directed to mammalian neurofilament triplet protein, and they are both stained by the modified Bodian method for SDS-PAGE (P. F. Drake and L. L. Phillips, manuscript in preparation). The Bodian staining method appears to be specific for neurofilament proteins from a wide variety of organisms, including molluscs (L. L. Phillips, R. J. Lasek, and L. AutilioGambetti, submitted for publication). These observations strongly indicate that the 60,000 -dalton and 65,000dalton proteins are major subunits of Aplysia NFs. Therefore, we will subsequently refer to these proteins as NF60 and NF65 to denote their relationship to the NFs. It is noteworthy that NF60 and NF65 are absent from the axonal connective sheath (Fig. $2 A$ ). This observation is consistent with the general observation that NF proteins are not expressed in non-neuronal cells.

To resolve the proteins in the cellular fractions further, we analyzed the proteins of the cell bodies and axons by two-dimensional SDS-PAGE. Figure 3 compares the proteins from the unextracted cell bodies and axons with those proteins which remain stably associated with the $\mathrm{Ti}$ fractions. Actin, tubulin, and the NF proteins are clearly resolved from one another by this gel system. Furthermore, the increased resolution provided by the isoelectric focusing step in two-dimensional SDS-PAGE separates NF65 into at least three separate highly insoluble subcomponents with different isoelectric points (see unextracted axons and cell bodies, and detergent-extracted cell bodies). The streaking of the NF60 from unextracted cell bodies is not a reproducible phenomenon and results when a large amount of protein is loaded on the gel.

The relative amount of NF60 and NF65 in axons appears to be substantially greater than in the cell bodies (Fig. 3). To verify these differences, proteins separated by one-dimensional SDS-PAGE were subjected to quantitative densitometry. In Table I the amount of each 


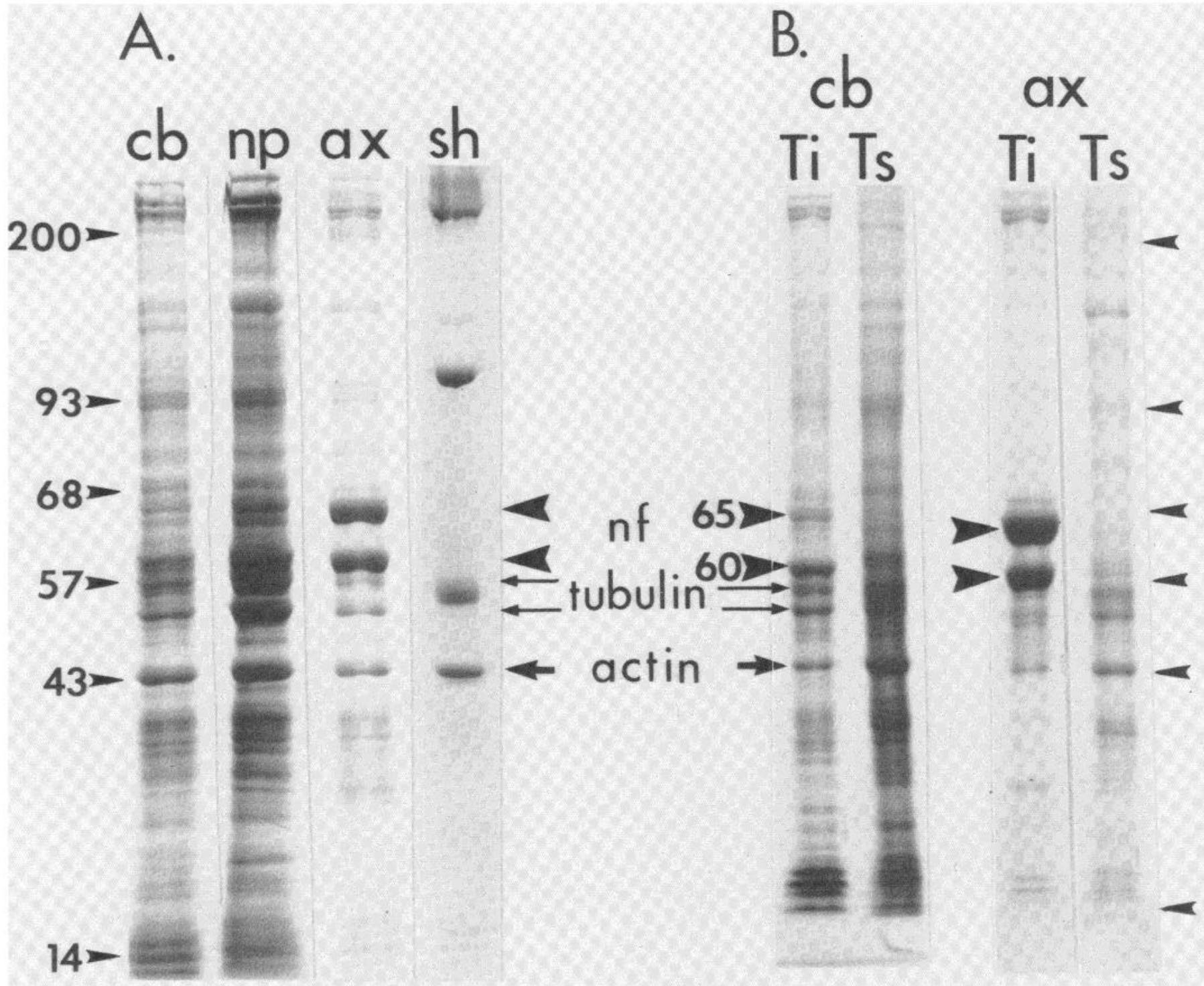

Figure 2. Regional distribution of neuronal proteins analyzed by CBB staining of one-dimensional SDS-PAGE. $A$, SDS-PAGE of proteins from populations of cell bodies $(c b)$, neuropile $(n p)$, desheathed axon-containing connectives $(a x)$, and connective sheath $(s h)$. Note the predominance of the NF proteins in the axon fraction. $B$, SDS-PAGE comparison of the Triton-insoluble (Ti) and Triton-soluble (Ts) fractions from cell bodies and axons. The NF proteins are insoluble, whereas tubulin and actin have both insoluble and soluble forms.

TABLE I

Quantitation of cytoskeletal proteins in cell bodies and axons

This table summarizes the quantitation of major cytoskeletal proteins from densitometric scans of gel profiles from 12 cell body and axon fractions, 4 animals, and 5 separate gels.

\begin{tabular}{lcc}
\hline & Cell Bodies & Axons \\
\hline & $\%$ total & \% total \\
Actin & $5.2 \pm 0.4$ & $5.8 \pm 0.6$ \\
$\beta$-Tubulin & $5.3 \pm 0.7$ & $5.6 \pm 0.5$ \\
$\alpha$-Tubulin & $3.4 \pm 0.9$ & $3.5 \pm 0.4$ \\
NF60 & $4.4 \pm 0.2$ & $16.9 \pm 1.2$ \\
NF65 & $2.7 \pm 0.3$ & $18.7 \pm 1.1$ \\
Total NF proteins & $6.9 \pm 0.5$ & $35.6 \pm 1.3$ \\
\hline
\end{tabular}

cytoskeletal protein is expressed as a percentage of the total protein. The cell bodies and axons contain similar percentages of actin, $\alpha$-tubulin, and $\beta$-tubulin. By contrast, the axons contain about 5 times more of the NF proteins than the cell bodies. This compositional difference between the $\mathrm{Ti}$ cytoskeletons of cell bodies and axons is also marked by a difference in the ratio of NF60 to NF65. The cell bodies contain proportionally more NF60 than the axons.

Additional differences between the cytoskeletal proteins of the cell bodies and axons were found by comparing their relative solubility. In Table II the relative amount of each protein which remained associated with the Ti fraction is presented as a percentage of the amount of that protein present in the $\mathrm{Ti}$ and $\mathrm{Ts}$ fractions combined. In the cell bodies, a large amount of tubulin remained associated with the Ti fraction. That is, nearly twice as much $\alpha$ - and $\beta$-tubulin remains bound to the insoluble framework in the cell body when compared to the axon. This difference between the cell bodies and axons is apparently specific for tubulin because there 


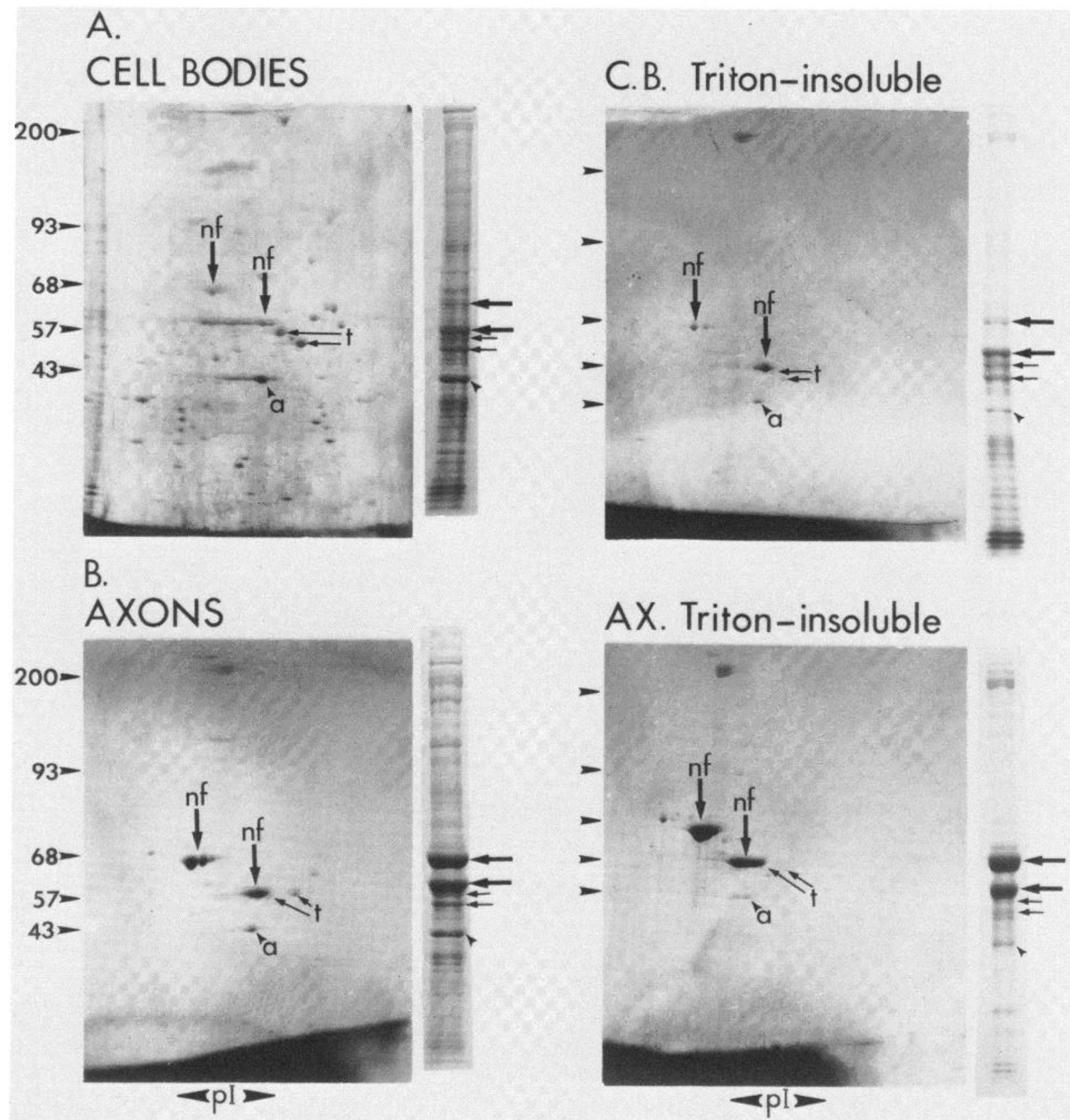

Figure 3. Two dimensional SDS-PAGE comparison of cell body and axonal proteins. $A$, SDS-PAGE representation of total and Triton-soluble cell body protein. $B$, SDS-PAGE profile of total and Trition-insoluble axonal proteins. Note the comparative abundance of the axonal NF ( $n f$ ) proteins. Tubulin $(t)$ and actin $(a)$ are marked by appropriate arrows and arrowheads on the one-dimensional gel lanes.

TABLE II

Stable forms of cytoskeletal proteins in cell bodies and axons This table summarizes the quantitation of major cytoskeletal proteins from densitometric scans of gel profiles from $10 \mathrm{Ti}$ and $\mathrm{Ts}$ cell body and axon fractions, 6 animals, and 5 separate gels.

\begin{tabular}{lll}
\hline & Cell Bodies & Axons \\
\hline & $\%$ Stable & \% Stable \\
Actin & $32.9 \pm 3.0$ & $26.6 \pm 3.2$ \\
$\beta$-Tubulin & $32.8 \pm 2.0$ & $18.8 \pm 1.6$ \\
$\alpha$-Tubulin & $43.8 \pm 5.4$ & $17.3 \pm 3.8$ \\
NF60 & 95 & 95 \\
NF65 & 95 & 95 \\
\hline
\end{tabular}

were no differences between the relative amounts of either stable actin or NF proteins when cell bodies were compared with axons (Table II).

Ultrastructure of isolated cell bodies and axon-containing connectives. The cytoskeletal proteins which remain stably associated with the $\mathrm{Ti}$ fractions are thought to represent stable polymeric forms of the proteins, such as MTs and NFs. To evaluate this possibility, we analyzed the structure of the cytoskeleton in individual cell bodies and desheathed connectives by electron microscopy. Preparations were analyzed either before or after extraction in the presence of Triton X-100. The morphology 
of $\mathrm{R} 2$, which is a representative neuron, is shown in Figure $4 A$. The cell body is marked by a large irregularly shaped nucleus and dramatic glial invaginations which often obscure the delineation of the plasma membrane. The juxtanuclear area and cortical cytoplasmic region are shown at higher magnifictions. The nucleus in Figure $4 B$ is delineated by a convoluted nuclear membrane and large nucleoli. The juxtanuclear cytoplasm is marked by a meshwork of ribosomes, mitochrondria, rough and smooth endoplasmic reticulum, and electron-dense regions. The cortical cytoplasm (Fig. $4 C$ ) is packed with pigment granules, and ribosomes are less abundant.

Triton extraction of an isolated cell body is accompanied by swelling of the cell, which is evident in the low magnification in Figure $4 D$. The nucleus is swollen and oval with vacuous areas which stand out in contrast to diffuse darkly stained areas. The effects of the extraction process are especially evident in the cortical cytoplasm where the intensity of staining with toluidine blue is significantly reduced in specimens prepared for light microscopy. The nuclear envelope in the extracted cell body is regular with less infolding, and the nucleus is less electron dense and the nucleoli more dispersed (Fig. $4 E$ ). The punctate nature of the juxtanuclear cytoplasm results from ribosomes and polysomes, and the vacuous spherical areas are the remnants of the pigment granules. The cortical cytoplasm is marked by these pigment granule remnants and by elongate aggregates of ribosomes (Fig. $4 F$ ).

A higher magnification of the juxtanuclear region and cortical cytoplasm from unextracted and detergent-extracted cell bodies is shown in Figure 5. The amount of flocculent material in the extracted cell bodies is reduced, resulting in a more open appearance. Although the biochemical data indicate that the soluble proteins have eluted after Triton extraction, the morphology of the insoluble preparations reveals the presence of intact organelles, such as mitochondria. This finding is not generalizable to all cytoplasmic organelles, as the integrity of the pigment granules is significantly altered after detergent extraction (see Fig. $4 F$ ). The presence of intact intracellular organelles is consistent with the observation that a cortical lamina composed of remnant membrane protein remains after Triton extraction (Ben-Ze'ev et al., 1979). Polysomes and monosomes are prominent structures of the $\mathrm{Ti}$ preparations and the appearance of MTs and abundant NFs is notable in the detergent-extracted preparations.

The desheathed pleural-abdominal connectives are dominated by axons of varying diameter and by projections of darkly stained glial cells (Fig. $6 A$ ). The inclusions in a single axon from the connectives are shown at higher magnification in Figure $6 B$. The presence of numerous axonal NFs and MTs is documented in Figure $6 C$. The detergent extraction has an effect on the axons, similar to that seen in the cell bodies (Fig. 6D). After extraction, the axons are marked by the presence of NFs and MTs as well as intact mitochondria and endoplasmic reticulum (Fig. 6, $E$ and $F$ ). The apparent abundance of the MTs and NFs in the axonal fractions is a result of the regular orientation of these polymeric structures in this region of the neuron. These electron microscopic obser- vations demonstrate that many MTs and NFs remain in the Triton-extracted preparations, suggesting that much of the tubulin and NF protein that remain associated with the Ti fractions are present in stable polymers.

\section{Individual cell and axon studies}

SDS-PAGE comparison of ${ }^{125}$ I-labeled cytoskeletal proteins in individual cell bodies and axons. To detect the small amounts of protein that are present in a single cell body or axon, we labeled the proteins with the [ $\left.{ }^{125} \mathrm{I}\right]$ Bolton-Hunter reagent. Although the Bolton-Hunter method has been shown to provide representative labeling of cytoplasmic proteins, we checked this method on Aplysia neurons by comparing with the more conventional CBB staining method. To have sufficient material for CBB staining, we used populations of cell bodies and desheathed connectives. The protein patterns observed with both methods are similar (Figs 2 and 7). However, some differences were found in the proteins revealed by these two methods. For example, three proteins of the axonal fraction in the molecular weight range of 14,000 to 30,000 daltons are more heavily labeled with BoltonHunter than with Coomassie blue. Also, a prominent perikaryal cytoskeletal protein at 18,000 daltons is detected with the Bolton-Hunter labeling method but not with the CBB method. This protein is highly insoluble (Fig. 7, large arrowheads) and is located exclusively in the cell body fraction.

Although we found some differences between the Bolton-Hunter and CBB protein profiles, the results of the two methods were comparable for the major cytoskeletal proteins which are the primary subject of this analysis. For example, the NF proteins are the dominant components of the Ti fraction, and the amounts of NF60 and NF65 are greater in the fraction enriched with axons than in the cell body fraction. These results confirm previous studies which have suggested that the BoltonHunter reagent provides a useful method for analyzing small amounts of proteins from complex cells and tissues (Katz et al., 1982). Therefore, we proceeded with our analyses of the proteins of individual cell bodies and their axons.

${ }^{125}$ I-labeled proteins from a single Triton-extracted R2 cell body and its attached axon are shown in Figure $7 B$. In these experiments the extracted and labeled neurons were further separated into cytoplasm, nucleus, and proximal axon. The overall labeling pattern for the cytoplasm in the cell body is similar to that of the axon. The major proteins of the cell body and axon are actin, the NF proteins, and the high molecular weight doublet fodrin (Bennett et al., 1982; Levine and Willard, 1982). A few proteins were found only in the cytoplasm of the cell body and not in the Ti fraction of the axon. Among these specific perikaryal proteins is the heavily labeled protein at 18,000 daltons. Also, note that the increase in the ratio of the NF60/NF65, illustrated in Figure 2, is not apparent in the initial $500-\mu \mathrm{m}$ axonal segment. This appears to be related to the processing of the NF proteins as they move from the cell body through the axon (P. F. Drake and R. J. Lasek, manuscript in preparation).

With the individual cells, it was possible to remove the nucleus and compare its protein composition with the 

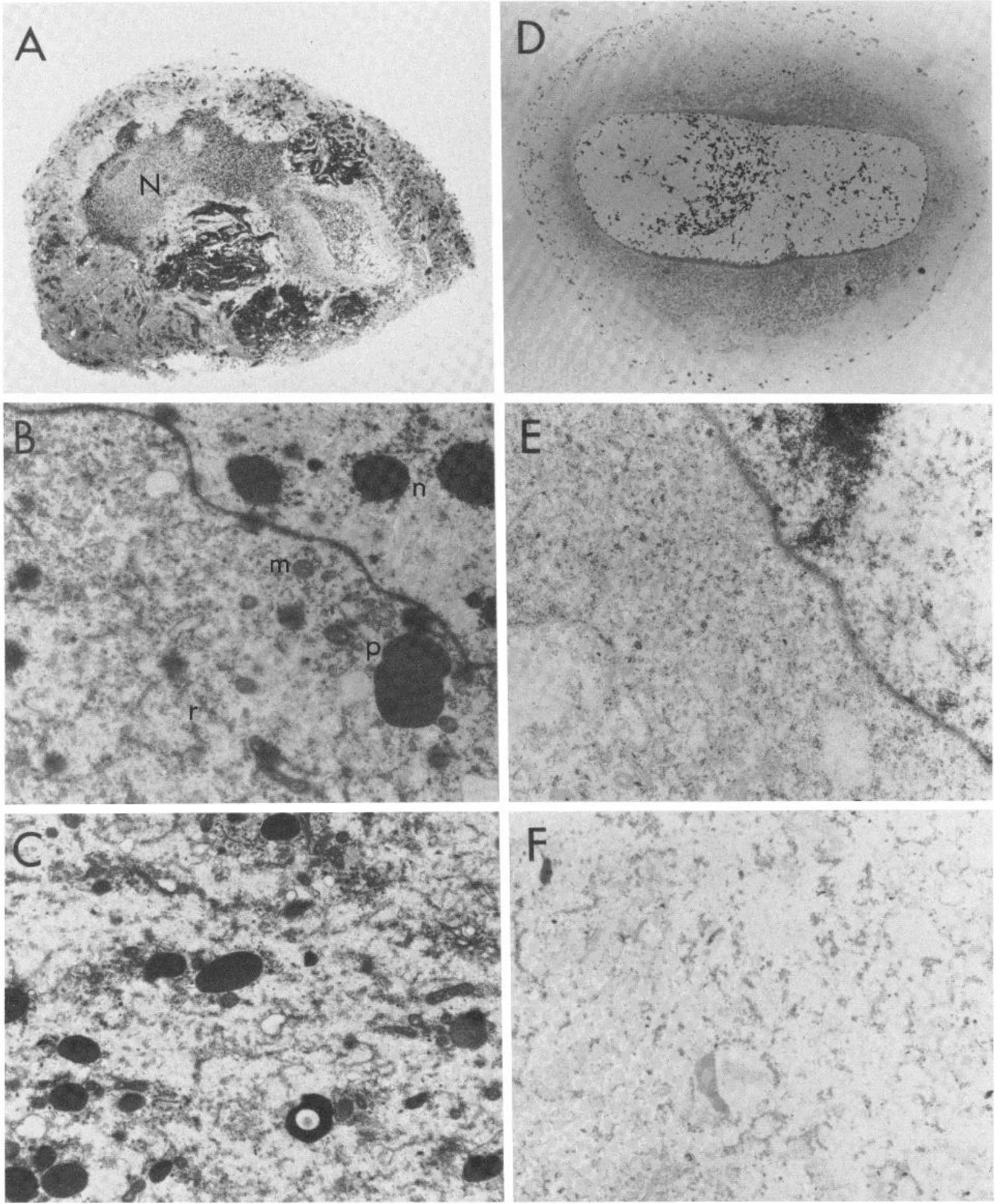

Figure 4. Ultrastructural comparison of unextracted ( $A$ to $C$ ) and Triton-extracted ( $D$ to $E$ ) R2 cell bodies. $A$, Unextracted cell body with large irregular nucleus $(N)$. Magnification $\times 250$. B, Micrograph of juxtanuclear area with cytoplasm containing pigment granules $(p)$, mitochondria $(m)$, and rough endoplasmic reticulum $(r)$. Nucleoli $(n)$ are prominent organelles in the nucleus. Magnification $\times 10,500$. $C$, Cortical cytoplasm with numerous pigment granules and electron-dense regions. Magnification $\times 10,500$. $D$, Triton extraction is accompanied by swelling of the cell. Magnification $\times 250$. $E$, The nucleus is less electrondense with dispersed nucleoli, and the nuclear envelope is regular with infolding. Remnants of the pigment granules are evident in the juxtanuclear cytoplasm, as are numberous monosomes and polysomes. Magnification $\times 10,500$. $F$, The cortical cytoplasm is significantly less electron-dense with pigment granule remnants and elongate aggregates of ribosomes. Magnification $\times 10,500$. 

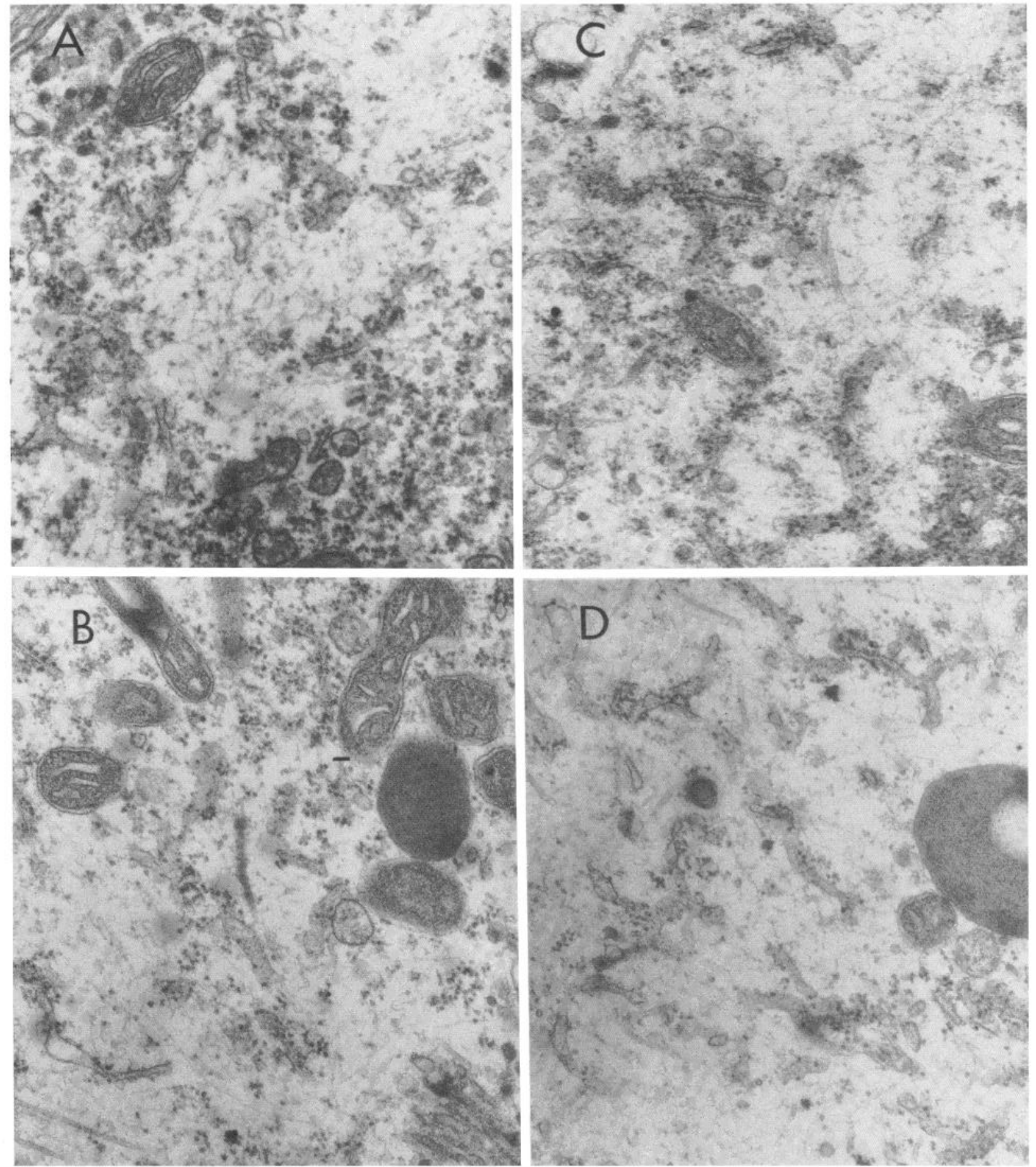

Figure 5. Ultrastructural comparison of perinuclear and cortical cytoplasm of unextracted and Triton-extracted R2 cell bodies. $A$, Unextracted cell body at perinuclear region. The presence of polysomes, mitochondria, rough endoplasmic reticulum, and a matrix of cytoskeletal polymers is notable. Magnification $\times 45,000$. B, Extracted cell body at perinuclear region. Intact cytoplasmic organelles, such as mitochondria and polysomes, are present; a meshwork of NFs and MTs is evident. Magnification $\times 45,000 . C$, Unextracted cell body at cortical cytoplasm is marked by a decrease in the density of polysomes. Magnification $\times 45,000$. $D$, Extracted cell body of cortical cytoplasm is marked by an open appearance with the major organelles consisting of rough endoplasmic reticulum, NFs, and MTs. Magnification $\times 45,000$.

cytoplasmic remnant. Several labeled proteins were found specifically in the nucleus; these nuclear proteins include the heavily labeled bands at 35,000 daltons and a series of three proteins in the molecular weight range of 70,000 to 150,000 daltons. However, the cytoskeletal proteins are not as prominent in the nucleus as in the cytoplasm. In fact, actin is the only cytoskeletal protein that is present in abundance in the nucleus (Fig. $7 B$, unpublished results from two-dimensional SDS-PAGE). Tubulin and the NF60 and NF65 are present almost 

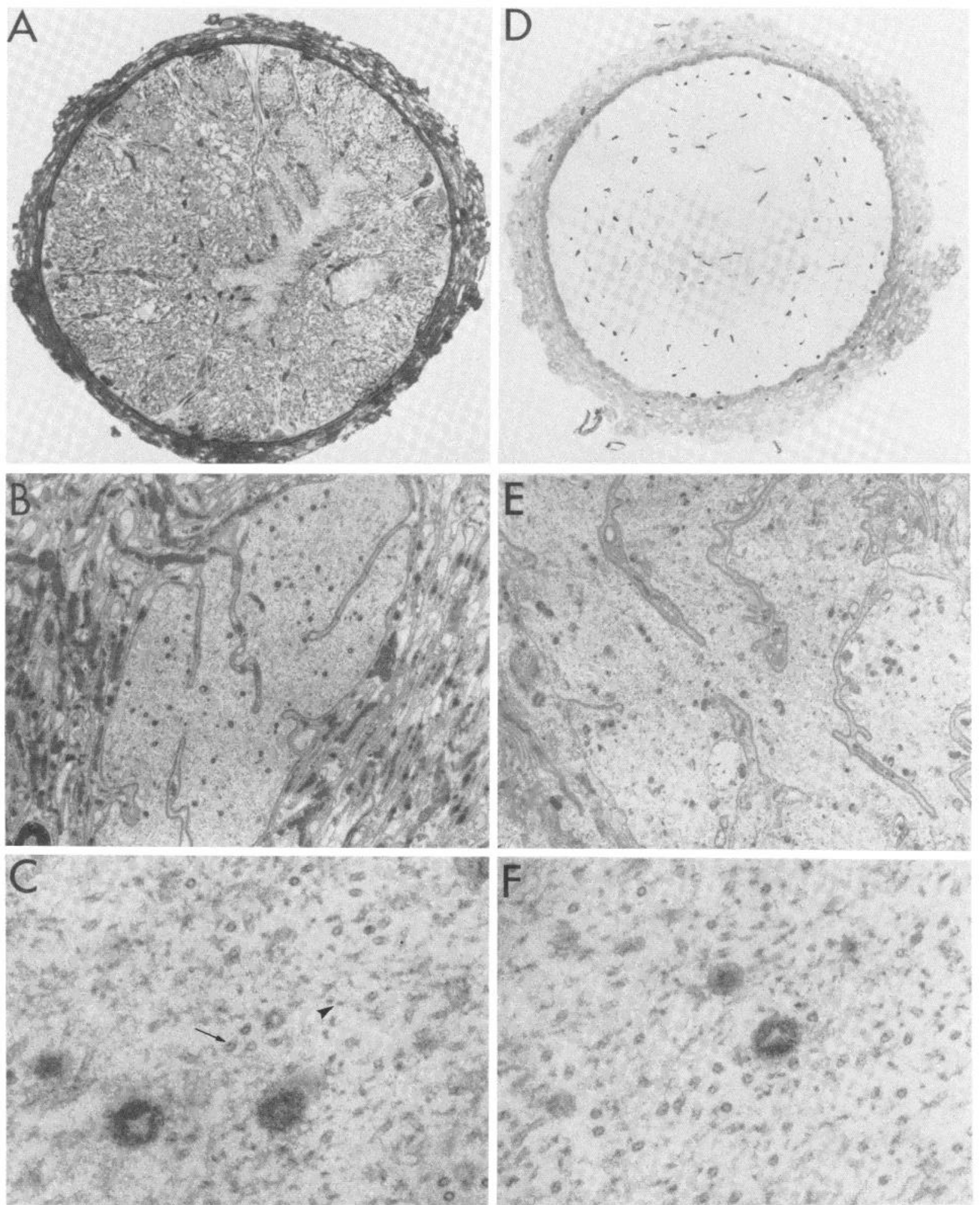

Figure 6. Ultrastructural comparison of unextracted axons ( $A$ to $C$ ) and Triton-extracted axons $(D$ to $F)$ from desheathed connectives. $A$, Connective filled with axons of varying size and shape. A remnant of the cortical sheath layer is evident. Magnification $\times 460$. $B$, Single axon within connective. Glial invaginations of the axonal membrane are evident. Magnification $\times 15,600$. C Cytoplasm from single axon with numerous MTs (arrow) and NFs (arrowhead) embedded in a floccular matrix. Magnification $\times 57,400$. $D$, Triton-extracted connective. The extent of extraction process is noted by reduced toluidine blue staining. Magnification $\times 460$. $E$, Single axon from extracted connective. Magnification $\times 15,600$. $F$, Cytoplasm from single extracted axon illustrating intact filamentous network including MTs and NFs. Magnification $\times 57,400$. 


\section{I BOLTON HUNTER}

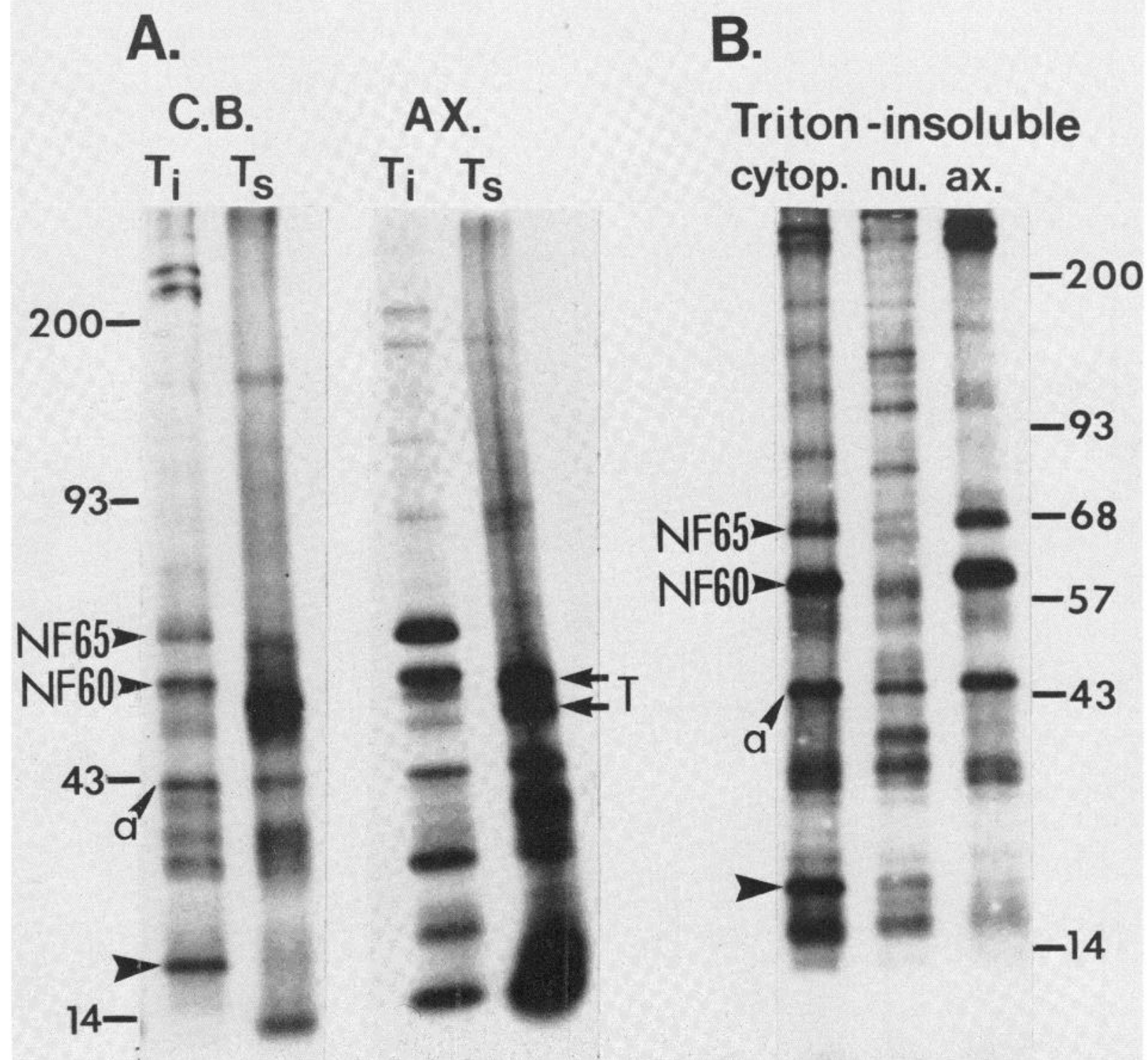

Figure 7. SDS-PAGE of cytoskeletal proteins from cell bodies and axons labeled with the Bolton-Hunter reagent. A, Comparison of Triton-insoluble $\left(T_{i}\right)$ and Triton-soluble $\left(T_{s}\right)$ proteins from populations of cell bodies $(C . B$. $)$ and desheathed axons $(A X$.). The NF proteins are insoluble while actin $(a)$ and tubulin $(T)$ have both soluble and insoluble forms. Note that the 18,000-dalton protein (arrowhead) is insoluble and specific to the cell body. B, Comparison of Ti proteins from a single cell body and axon. After extraction and labeling, the nucleus ( $n u$.) and proximal axon were isolated from the cell body remnant. Actin $(a)$ and the $\mathrm{NF}$ proteins are evident in the cytoplasm (cytop.) and proximal 500- $\mu \mathrm{m}$ segment of axon $(a x)$. The 18,000-dalton protein (arrowhead) is cytoplasm specific, and actin is present in the nucleus.

exclusively in the cytoplasm. These results indicate that the nucleus contains very little tubulin or NF protein and that these proteins are present in the cytoplasm. Thus, it is not necessary to remove the nucleus to obtain a valid estimate of the cytoskeletal composition of Aplysia neurons.

In summary, these results demonstrate that the composition of the cytoskeleton in Aplysia neuron can be analyzed by either the CBB method or the BoltonHunter method. Comparisons of the results obtained from individual neurons and their axons with those from populations of cells and desheathed connectives indicate that the more complex samples provide a useful index of the composition of the neuronal cytoskeleton.

\section{Discussion}

The proteins in the disssected cell bodies and axons are primarily neuronal. Our results demonstrate that the composition and biochemical properties of the cytoske- 
letal proteins in the neuron cell bodies differ from those associated with axons in the nervous system of Aplysia. To relate these findings specifically to the composition of the neuron, we must be certain that no other components, such as glia, contribute a significant amount of proteins to these preparations. Although these neuronal preparations were carefully dissected to remove any contaminating material, we did not remove the thin layer of glial cells which tightly invests the cell bodies and axons. Morphological analyses demonstrate that the adherent glial cells represent a comparatively small amount of material in any of these fractions (Figs. 4 and 6). Moreover, comparisons of the proteins which are synthesized by the glial cells and those synthesized by the neuron demonstrate dramatic differences; glial proteins bear little resemblance to those proteins found in the cell bodies (P. Drake and R. J. Lasek, manuscript in preparation). By contrast, the proteins specifically synthesized by the nerve cell bodies correlate very well with the proteins detected in this study. Taken together, these results indicate that the glia contribute very little protein to the fractions that we have analyzed. This also appears to be the case for the axons in the desheathed connectives which have a similar composition to the individual axons that were microdissected from the ganglia. We conclude that the proteins analyzed in our study are primarily neuronal in origin.

Regional differences in the composition of the cytoskeleton. The ultrastructural data indicate that under our buffer conditions, extraction with Triton X-100 does not significantly disrupt the basic organization of the neuronal cytoplasm. The structural integrity of many cytoplasmic organelles, including polysomes, rough endoplasmic reticulum, mitochondria, and the Golgi complex, is preserved. The overall morphology of these extracted neurons is similar to that of extracted cells in tissue culture, where the presence of the cytoskeletal network (MTs and NFs) serves to conserve cyloplasmic oryanization (Lenk et al., 1977; Ben-Ze'ev et al., 1979). A significant proportion of the total cytoskeletal proteins remains associated with the extracted cell bodies and axons, and comparison of the cytoskeletal proteins in these different regions of the neuron reveals major compositional differences. Specifically, the NF60 and NF65 are 5 times more abundant in the axon than in the cell body, whereas the relative amounts of actin and tubulin are comparable in these two regions of the neuron. Stated another way, the ratio of total NF protein to tubulin is greater in the axon than in the cell body. Although no comparable quantitative biochemical analyses of the cytoskeleton exist for vertebrate neurons, immunocytochemical studies of vertebrate neurons suggest that the $\mathrm{NF}$ content of the axon is higher than that of the cell body (Shaw et al., 1981). The ratio of NFs to MTs is of interest because MTs and NFs are the principal components of the cytoskeleton, and these two types of polymers are structurally linked by cross-bridges to form the backbone of the cytoskeleton (Lasek, 1980). In many types of vertebrate axons, tubulin and the NF proteins are transported coordinately in slow axonal transport as part of a proposed MT/NF network (Lasek, 1980).

Another striking example of regional differences be- tween the cytoskeletal proteins in the cell body and axons was found in the composition of the NF subunits. The ratio of NF60 to NF65 is greater in the cell body than it is in the axon where NF65 predominates. In addition, the physical properties of the tubulin in the cell body differ from that in the axon (Table II). The cell bodies contain twice as much insoluble tubulin as the axons. This insoluble tubulin is probably in the form of stable MTs which do not depolymerize because the dissociation reaction is blocked at both ends of the polymer (Lasek and Morris, 1982; Morris and Lasek, 1982). It is interesting that analyses of the cytoskeleton in the squid giant axon also demonstrate that approximately $17 \%$ of the tubulin is in the form of stable polymer (Lasek and Morris, 1982). The similarity of these results suggests that the observations made on Aplysia neurons apply more generally.

Aplysia neurons may have two cytoskeletal networks. The substantial difference between the composition of the cytoskeleton of the neuron cell bodies and axons suggests that these neurons have at least two distinct cytoskeletal networks. To understand the mechanisms that differentiate these cytoskeletal networks it is important to recall that all of the cytoskeletal proteins of the neuron are synthesized in the cell body and that the axonal proteins are transported from the cell body into the axon. Two possible mechanisms exist which could differentiate the axonal cytoskeleton from that of the cell body. One mechanism, which we will call "differential organization," involves the spatial segregation of the two cytoskeletal networks in the cell body. Differential organization implies that there are two separate organizing centers for the organization of the cytoskeletal networks, one for the cell body and another for the axon. The segregation between these two organizing centers could occur at the level of protein synthesis or assembly. The other possible mechanism, which we will call "differential metabolism," involves the selective metabolism of the axonal cytoskeleton after it is transported from the cell body into the axon. That is, the cell body may contain a single homogeneous cytoskeletal network from which the axonal cytoskeletal elements are drawn. Differences in the axonal cytoskeleton might then be produced after the cytoskeletal elements are transported from the cell body into the axon. These two mechanisms are not mutually exclusive.

Differential metabolism of the cytoskeleton as it moves along the axon has been demonstrated. For example, the NFs in Aplysia undergo a post-translational modification during their transit through the axon (P. F. Drake and R. J. Lasek, manuscript in preparation). Mammalian NF proteins also undergo a minor proteolytic modification during their transport through the retinal ganglion cell axons (Nixon et al., 1982). $\Lambda$ substantial amount of evidence suggests that differential organization is also an important mechanism in segregating the cytoskeletal networks of the neurons. Dorsal root ganglion cells provide the most dramatic evidence supporting the differential organization mechanism. The cytoskeletons of the central and peripheral branches of these cells have very different compositions and transport properties (Mori et al., 1979). Furthermore, the cell body is apparently ca- 
pable of specifically altering the properties of the cytoskeleton that are transported into one branch in response to axotomy of that branch (Oblinger and Lasek, 1983).

In the case of the Aplysia neuron, we believe that the striking differences in the ratios of NF proteins to tubulin between cell body and axon can most readily be explained by the mechanism of differential organization. Many questions remain regarding the mechanism of differential organization of the cytoskeleton. For example, differential organization implies that there are separate organizing centers for the different cytoskeletal networks. It will be important to determine whether organizing centers also involve a regional segregation of the protein synthetic machinery. That is, there may be two separate groups of polysomes which synthesize proteins exclusively for each network.

Regional differences in the cytoskeleton of neurons. Our observation that the Ti cytoskeleton of the cell body is different from that of the axon raises questions about the functional consequences that these differences have in the neuron. The most obvious function of the cytoskeleton is its role in providing structural support and in defining cell shape. The cylindrical shape of the axon is completely different from the spherical shape of the cell body, and the cytoskeletal elements must be very important in generating these shapes. How might the differences that we have found in the composition of the cytoskeleton be related to the differences in the shape of the cell body and axon? In the adult neuron, the cytoskeleton maintains the pre-existing form which was established during development. In this regard, it is interesting that the axonal cytoskeleton has a much higher content of NFs than the cytoskeleton in the cell body. The NFs appear to be principally structural in function. They are members of the larger family of intermediate filament proteins, and one of the hallmarks of this class of proteins is their relative insolubility in physiological solutions. The most extreme example of these solubility properties are the $\alpha$-keratins, which are the subunits of keratin filaments in hair and skin. It seems likely that one of the functions of the NFs in the axonal cytoskeleton is to add stability to axonal structure.

NFs may function to increase the diameter of axons. Another important function of the NFs is their role in determining the volume of the cytoskeleton. NFs are interconnected by numerous cross-links which both stabilize the cytoskeletal network and separate the NFs from each other. Although the NFs are only $10 \mathrm{~nm}$ in diameter, they displace a much larger volume because of their side arms. Furthermore, the NFs may fill space at relatively small metabolic expense as compared to MTs. For example, the regions of the cytoplasm occupied by NFs appear to be much simpler in structure, and probably in chemical composition, than the regions occupied by MTs. MTs are surrounded by a rich feltwork of proteins corresponding to the axoplasmic matrix. The matrix apparently includes a wide variety of metabolic enzymes and regulatory proteins (Brady and Lasek, 1981). Thus, the MTs define regions of the cytoplasm that are metabolically complex and probably very active. By contrast, the NF-containing regions are simple and may be metabolically inert.

Studies of axons during development also indicate that
NFs may function as additional supporting components to the more metabolically active MT domains and elements of the cytomatrix. The cytoskeletons of embryonic axons consist principally of MTs. As the axons grow in diameter, NFs are added in increasing numbers, and there is a direct relationship between cross-sectional area of axons and the number of NFs in the axon (Friede and Samorajski, 1970). These observations suggest that NFs function in great part to increase locally the volume of the cytoskeleton. Large axons conduct action potentials more rapidly than small axons. Large, rapidly conducting axons have evolved independently in many different animal forms. The axonal cytoskeleton of these large axons usually contains a large proportion of NFs.

On the basis of these various observations, we propose that the difference between cytoskeletons of the axon and of the cell body are related to the differences in the requirements of their size. MTs are required in both the cell body and axon because of their important role in organizing the movement of membrane traffic. On the other hand, the addition of large numbers of NFs to the axonal cytoskeleton may serve to selectively increase the diameter of the axon. Furthermore, the NFs act to stabilize the long and often complex shape of the axon. These two roles may be among some of the important functional differences that are generated by regional differentiation of the cytoskeleton. It will be of great interest to obtain additional information about the compositional differences of the cytoskeletons in different parts of other neurons (such as dendrites) to compare with the information that we have found for Aplysia neurons.

\section{References}

Baumgold, J., S. Terakawa, K. Iwasa, and H. Gainer (1981) Membrane-associated cytoskeletal proteins in squid giant axons. J. Neurochem. 36: 759-764.

Bennett, V., J. Davis, and W. E. Fowler (1982) Brain spectrin, a membrane-associated protein related in structive and function to erythrocyte spectrin. Nature 299: 126-131.

Ben-Ze'ev, A., A. Duerr, F. Solomon, and S. Penman (1979) The outer boundry of the cytoskeleton: A lamina derived from plasma membrane proteins. Cell 7: 859-866.

Bolton, A. E., and W. M. Hunter (1973) The labelling of proteins to high specific radioactivities by conjugation to a ${ }^{125} \mathrm{I}$-containing acylating agents. Biochem. J. 133: 529-539.

Bonner, W. M., and R. A. Laskey (1974) A film detection method for tritium labelled proteins and nucleic acids in polyacrylamide gels. Eur. J. Biochem. 46: 83-88.

Brady, S. T., and R. J. Lasek (1981) Nerve-specific enolase and creatine phosphokinase in axonal transport: Soluble proteins and the axoplasmic matrix. Cell 23: 515-523.

Fink, D. J., and H. Gainer (1979) The use of a labeled acylating probe for the study of fast axonal transport, in vivo. Brain Res. 117: 208-213.

Fink, D. J., and H. Gainer (1980) Axonal transport of proteins: A new view using in vivo covalent labelling. J. Cell Biol. 85 : $175-186$.

Friede, R. L., and T. Samorajski (1970) Axon caliber related to neurofilaments and microtubules in sciatic nerve fibers of rats and mice. Anat. Rec. 167: 379-388.

Kandel, E. R. (1976) Cellular Basis of Behavior, W. H. Freeman, San Francisco.

Katz, M. J., R. J. Lasek, P. Osdoby, J. R. Whittaker, and A. I. 
Caplan (1982) Bolton-Hunter reagent as a vital stain for developing systems. Dev. Biol. 90: 419-429.

Laemmli, U. K. (1970) Cleavage of structural proteins during the assembly of the head of bacteriophage T4. Nature 277 : 680-685.

Lasek, R. J. (1980) Axonal transport: A dynamic view of neuronal structures. Trends Neurosci. 3: 87-91.

Lasek, R. J. (1981) The dynamic ordering of neuronal cytoskeletons. Neurosci. Res. Program Bull. 19: 7-32.

Lasek, R. J. (1982) Translocation of the neuronal cytoskeleton and axonal locomotion. Philos. Trans. R. Soc. Lond. Biol 299: 313-327.

Lasek, R. J., and J. R. Morris (1982) The microtubule-neurofilament network: The balance between plasticity and stability in the nervous system. In Biological Functions of Microtubules and Related Structures, pp. 329-341, Academic Press, New York.

Eur. J. Biochem. 56: 335-341.

Lenk, R., L. Ransom, Y. Kaufmann, and S. Penman (1977) A cytoskeletal structure with associated polyribosomes obtained from Hela cells. Cell 10:67-78.

Levine, J., and M. Willard (1981) Fodrin: Axonally transported polypeptides associated with the internal periphery of many cells. J. Cell Biol. 90: 631-643.

Matus, A., R. Bernhardt, and T. Hugh-Jones (1981) High molecular weight microtubules-associated proteins are pref- erentially associated with dendritic microtubules in brain. Proc. Natl. Acad. Sci. U. S. A. 78: 3010-3014.

Mori, H., Y. Komiya, and M. Kurokawa (1979) Slowly migrating axonal polypeptides. Inequalities in their rate and amount. J. Cell Biol. 82: 174-184.

Morris, J. R., and R. J. Lasek (1982) Stable polymers of the axonal cytoskeleton: The axoplasmic ghost. J. Cell Biol. 92: $192-198$.

Nixon, R. A., B. A. Brown, and C. A. Marotta (1982) Posttranslational modification of neurofilament protein during axoplasmic transport: Implications for regional specialization of CNS axons. J. Cell Biol. 94: 150-158.

Oblinger, M. M., and R. J. Lasek (1983) Effect of conditioninglesion on the rate of regeneration of two branches of the DRG cell. Anat. Rec. 205: 145a.

O'Farrell, P. H. (1975) High resolution of two-dimensional electrophoresis of proteins. J. Biol. Chem. 250: 4007-4021.

Palay, S. L., and V. Chan-Palay (1974) Cerebellar Cortex, Springer-Verlag, New York.

Rubinson, K. A., and P. F. Baker (1979) The flow properties of axoplasm in a defined chemical environment: Influence of anions and calcium. Proc. R. Soc. Lond. Biol. 205: 323-345.

Shaw, G., M. Osborn, and K. Weber (1981) An immunofluorescence study of the neurofilament triplet proteins, vimentin and glial fibrillary acidic protein within the adult rat brain. Eur. J. Cell Biol. 26: 68-82. 